www.nature.com/pj

\title{
Polymeric nanofibers: isodiametric design space and methodology for depositing aligned nanofiber arrays in single and multiple layers
}

\author{
Polymer Journal (2013) 45, 695-700; doi:10.1038/pj.2013.1; published online 13 February 2013
}

\section{INTRODUCTION}

Polymeric micro/nanofibers are increasingly gaining importance due to their versatile applications in diverse fields, such as tissue engineering, smart textiles, sensors and actuators. Traditionally, the well-established techniques of spun bounding, ${ }^{1,2}$ melt blowing, ${ }^{3,4}$ dry spinning, ${ }^{5,6}$ conjugate spinning ${ }^{7}$ and $\mathrm{CO}_{2}$ laser thinning ${ }^{8}$ produce polymeric fibers with diameters on the micron scale and lengths in excess of a meter. However, aligned deposition and continuous production of long fibers with nanoscale diameters has been a challenge due to the fragility of polymeric materials. In addition, numerous applications require the deposition of smooth, uniform and defect-free nanofiber arrays in aligned configurations with user-defined geometrical spacing. Several processes have been developed to fabricate polymer nanofiber arrays, including electrospinning, ${ }^{9-13}$ template synthesis, ${ }^{14,15}$ phase separation $^{16,17}$ and sequential micro dry spinning. ${ }^{18}$ Of all these methods, electrospinning is perhaps the most popular process, allowing for the continuous production of fibers ranging from tens of nanometers to a few microns in diameter and requiring specialized strategies for the aligned deposition of these fibers. ${ }^{19-24}$

Although nanofibers can be essentially aligned in single and multiple layers, tight control over the fiber diameter and spacing has yet to be realized. The deficiencies of the current state-of-the-art fabrication methods continue to hinder improvements in the applications of polymer nanofibers. Here, we present a non-electrospinning strategy based on our previously reported STEP (Spinneret based Tunable Engineered Parameters) technique ${ }^{25}$ to deposit arrays of polymer fibers with uniform diameters ranging from sub-100 $\mathrm{nm}$ to microns and lengths of at least several millimeters. In addition, this strategy has the ability to control adjacent fiber-fiber spacing. The control of fiber diameter is achieved by manipulating the polymer chain entanglements in the polymer-solvent solution, which are directly related to the polymer solution concentration and the molecular weight. The molecular entanglement-fiber diameter relationships are mapped to obtain an isodiametric design space, which provides a convenient visual tool for estimating the fiber diameters. Furthermore, nanofiber assemblies in single and multiple layers are demonstrated using isodiametric fibers as building blocks, which helps to establish a much needed, robust engineering framework for depositing aligned micro/nanofibers in hierarchical assemblies.

\section{EXPERIMENTAL PROCEDURE}

\section{Solution preparation}

All chemicals were used as received without further purification. Polystyrene (PS) of several molecular weights $\left(M_{W}\right)\left(650,860,1500\right.$ and $2000 \mathrm{~K} \mathrm{~g} \mathrm{~mol}^{-1}$, Scientific Polymer Products Inc., Ontario, NY, USA) were dissolved at room temperature in $p$-xylene with varying $\mathrm{wt} \%$ concentrations (from $1 \mathrm{wt}$ to $18 \mathrm{wt} \%$ ) for 1 week before experiments. Poly(methyl methacrylate) (PMMA, $M_{W}=540$ $\mathrm{Kg} \mathrm{mol}^{-1}$, Scientific Polymer Inc., Ontario, NY, USA) dissolved in chlorobenzene at $14 \mathrm{wt} \%$ and poly(lactic-co-glycolic acid) (PLGA, Purac Biochem, Gorinchem, Holland) dissolved in chloroform at $3 \mathrm{wt} \%$ were used in this study. Bovine plasma fibrinogen (Sigma-Aldrich Chemical, St Louis, MO, USA) was dissolved in a solution composed of 9 parts 1,1,1,3,3,3-hexafluoro-2propanol and 1 part $10 \times$ minimal essential medium at $20 \mathrm{wt} \%$ for 1 week before experiments. Polyurethane (PU5719, Lubrizol Advanced Material, Wickliffe, OH, USA) was dissolved in dimethylformamide at $30 \mathrm{wt} \%$ and heated to $80^{\circ} \mathrm{C}$ with stirring for $24 \mathrm{~h}$ to prepare a homogenous solution. One gram of titanium isopropoxide $\left(\mathrm{Ti}(\mathrm{O} i \mathrm{Pr})_{4}\right.$, Sigma-Aldrich Chemical) was mixed with $2.7 \mathrm{~g}$ of a $7 \mathrm{wt} \%$ PS $\left(M_{W}=2000\right.$ $\mathrm{K})$-xylene solution, followed by magnetic stirring for $1 \mathrm{~h}$ before experiments.

\section{STEP spinning}

The spinning process was performed at a temperature of $20^{\circ} \mathrm{C}$ and a relative humidity of $13-17 \%$. The viscous polymer solutions were pumped through the micropipette spinneret $(\mathrm{ID}=100 \mu \mathrm{m})$ and formed an extruded solution droplet at the exit (Figure 1a). The substrate was mounted on a DC motor, which, in turn, was mounted on a motorized micro-positioning stage with three degrees of freedom (VP-25XA, Newport Inc., Stanta Clara, CA, USA). After the substrate made contact with the solution droplet, the fibers were collected on the substrate in aligned configurations. No voltage source was used in the experiments to eject the polymer solution.

\section{Solvent treatment}

A fused PMMA fiber network was obtained by exposing the PMMA fiber assembly to chlorobenzene vapor at room temperature in an enclosed chamber for $20 \mathrm{~s}$.

\section{Calcination}

As-spun PS-titanium isopropoxide fibers were left in air for $4 \mathrm{~h}$ to allow for the complete hydrolysis of $\mathrm{Ti}(\mathrm{O} i \mathrm{Pr})_{4}$. PS was removed by treating the fibers in air at $750^{\circ} \mathrm{C}$ for $4 \mathrm{~h}$.

\section{Fiber dyeing}

Fluorescent reagents DiT (Red), DiD (Purple) and DiC (Green) (Biotium Inc., Hayward, CA, USA) were dissolved in dimethyl sulfoxide at $1 \mathrm{mg} \mathrm{ml}^{-1}$, and the solutions were sonicated for $5 \mathrm{~h}$ at $50^{\circ} \mathrm{C}$. For the fiber staining process, $1 \mu \mathrm{l}$ of fluorescent reagent solution was added to $1 \mathrm{ml}$ polymer solutions, and the mixture was further sonicated for $1 \mathrm{~h}$ before spinning. 

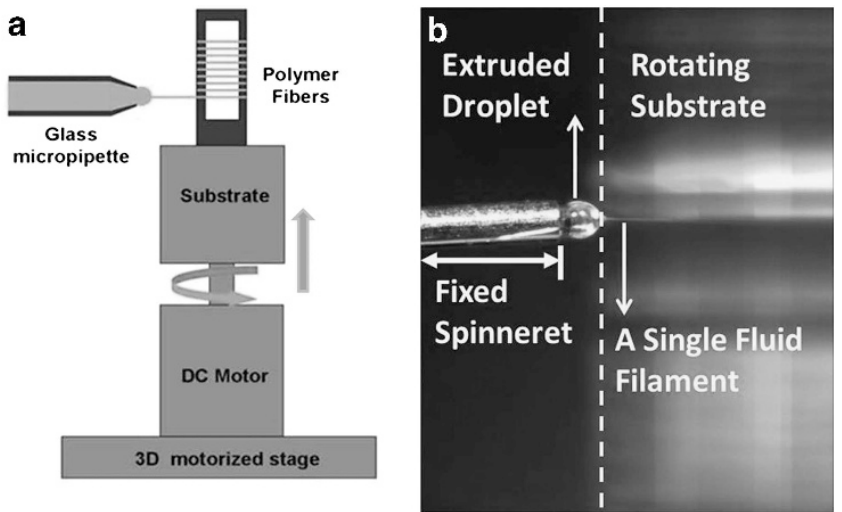

Figure 1 (a) A schematic illustration of the experimental set-up of the STEP technique; (b) an optical image of the fiber formation process with the edge of the rotating substrate represented by the dashed line. A full color version of this figure is available at Polymer Journal online.
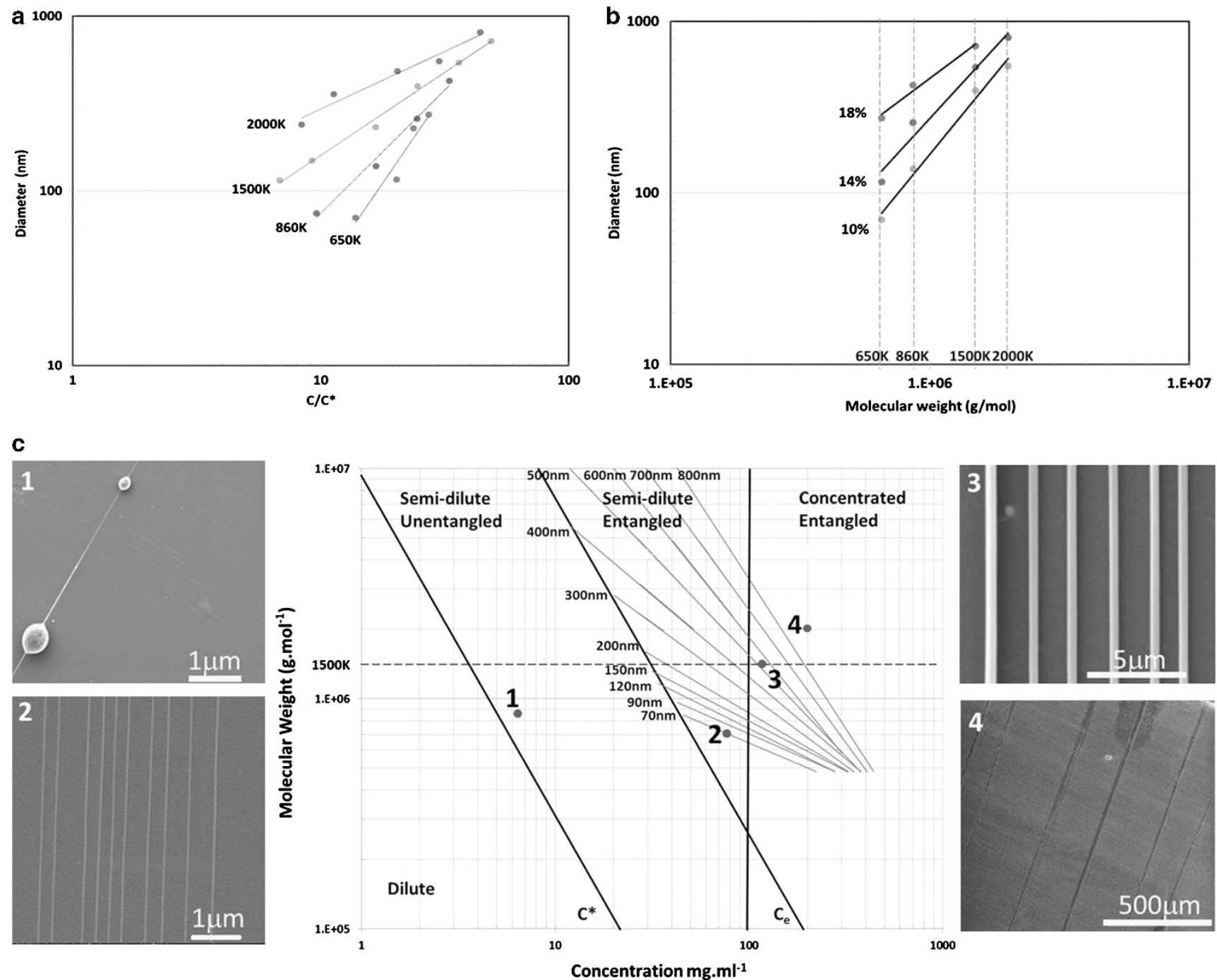

Concentration $\mathrm{mg}^{\mathrm{m} \mathbf{l}^{-1}}$

Figure 2 The linear regression analysis developed for the fiber diameter vs (a) the normalized concentration $\left(C / C^{*}\right)$, (b) the molecular weight and (c) the isodiametric design space for the PS nanofibers superimposed on the Graessley diagram: ${ }^{27}$ the blue lines represent isodiametric lines, and the numbers on these lines denote the fiber diameters. Inserts 1-4 are representative images corresponding to the four points (noted as 1-4) in the design space. Each data point in $\mathbf{a}$ and $\mathbf{b}$ is an average result of at least 50 measurements. A full color version of this figure is available at Polymer Journal online. 


\section{Characterization}

All measurements were performed at room temperature. The intrinsic viscosity and the MarkHouwink-Sakurada constants were obtained through dilute solution viscometry experiments (Supplementary Information, SI1). Field-emission scanning electron microscopy studies were conducted using an FEI Quanta 600 microscope working at $5-25 \mathrm{kV}$. Fluorescent images were taken with an Axio Observer (Carl Zeiss Microimaging Inc., Thornwood, NY, USA). Optical images were taken with an AmScope 800 digital camera attached to a $\times 30$ microscope (Carl Zeiss Microimaging Inc.).

\section{RESULTS AND DISCUSSION}

STEP-based fiber fabrication

The STEP technique requires an ambient atmosphere to evaporate the solvent, leading to the formation of a solid fiber, but more importantly, the technique does not require the aid of an electric field, as is the case with electrospinning. As shown in Figure 1b, a micropipette loaded with polymer solution is connected to a pressure source, which results in an extruded polymer solution droplet at the tip of the micropipette. A rotating substrate is brought into contact with the solution droplet, thus pulling out a single filament. During the stretching process of the solution filament, continual evaporation of the solvent causes an increase in molecular entanglements, thereby reducing the overall deformability. A solidified fiber is formed by rapid solvent evaporation and collected on the substrate in an aligned configuration. In contrast to electrospinning, which relies on an electric field, STEP relies on the rotating speed of the substrate to 'stretch and thin' the solution filament. We emphasize that for the STEP technique, the fluid filament is pulled out, rather than ejected, from the tip of the droplet and thus resembles a fluid element being pulled out from an endless reservoir. As no voltage is involved, the fluid jet whipping and spraying present in other methods are avoided in STEP manufacturing, resulting in parallel fiber arrays with narrow fiber diameter distributions.

Using the STEP technique, hierarchical fiber assemblies are easily achieved by depositing fiber arrays on top of previous layers at desired angles with user-defined fiber spacing. High aspect ratio fibers of long lengths $(\mathrm{mm}-\mathrm{cm})$ with diameters ranging from sub- $100 \mathrm{~nm}$ to microns (length/diameter aspect ratio > 2000) were obtained by optimizing the processing parameters (rotating speed of the substrate) and material parameters (solution concentration and polymer molecular weight). We have previously reported that an increase in the rotational speed of the substrate $\left(V_{\mathrm{r}}\right)$ leads to a decrease in the fiber diameter and that, for speeds exceeding $1 \mathrm{~m} \mathrm{~s}^{-1}$, constant diameter fibers were obtained. ${ }^{25}$ Furthermore, we also reported an increase in the fiber diameter with increased polymer solution concentration. In this study, we present an engineered design space at constant $V_{\mathrm{r}}$ $\left(1.2 \mathrm{~m} \mathrm{~s}^{-1}\right)$ for fabricating uniform diameter fibers $(70-800 \mathrm{~nm})$ with high aspect ratios $(>2000)$. The isodiametric design space for fabricating continuous fibers is dependent upon achieving a minimum number of polymeric molecular chain entanglements for different molecular weights. The entanglements in a polymer solution are typically represented by critical overlap concentrations $\left(C^{*}\right)$ and critical entangled concentrations $\left(C_{\mathrm{e}}\right)$ (Supplementary Information, SI1).

\section{Isodiametric design space}

PS was dissolved in $p$-xylene with increasing concentrations at four molecular weights (650, 860,1500 and $2000 \mathrm{~K} \mathrm{~g} \mathrm{~mol}^{-1}$ ). The diameter dependence on the solution concentration $(C)$ was then investigated (Figure 2a) and was found to scale with $C^{*}$ as:

$$
\begin{aligned}
& 650 \mathrm{~K}: \operatorname{Dia}(\mathrm{nm})=0.3\left(\frac{C}{C^{*}}\right)^{2.1} \\
& 860 \mathrm{~K}: \operatorname{Dia}(\mathrm{nm})=2.9\left(\frac{C}{C^{*}}\right)^{1.4} \\
& 1500 \mathrm{~K}: \operatorname{Dia}(\mathrm{nm})=10.3\left(\frac{C}{C^{*}}\right)^{1.1} \\
& 2000 \mathrm{~K}: \operatorname{Dia}(\mathrm{nm})=60.7\left(\frac{C}{C^{*}}\right)^{0.7}
\end{aligned}
$$

The scaling laws were developed using $C^{*}$, as the critical overlap concentrations were accurately obtained from the dilute solution intrinsic viscosity experiments. Similarly, the diameter dependence on the molecular weight for different concentrations was investigated (Figure 2b) and found to scale as:

$$
\begin{aligned}
& 10 \% \text { wt }: \operatorname{Dia}(\mathrm{nm})=1 \mathrm{E}-9\left(M_{w}\right)^{1.8} \\
& 14 \% \text { wt }: \operatorname{Dia}(\mathrm{nm})=4 \mathrm{E}-8\left(M_{w}\right)^{1.6} \\
& 18 \% \text { wt }: \operatorname{Dia}(\mathrm{nm})=8 \mathrm{E}-5\left(M_{w}\right)^{1.1}
\end{aligned}
$$

As evidenced by Equations (1)-(4), the lower molecular weight species exhibit higher exponents, which demonstrates that a higher concentration is required for the formation
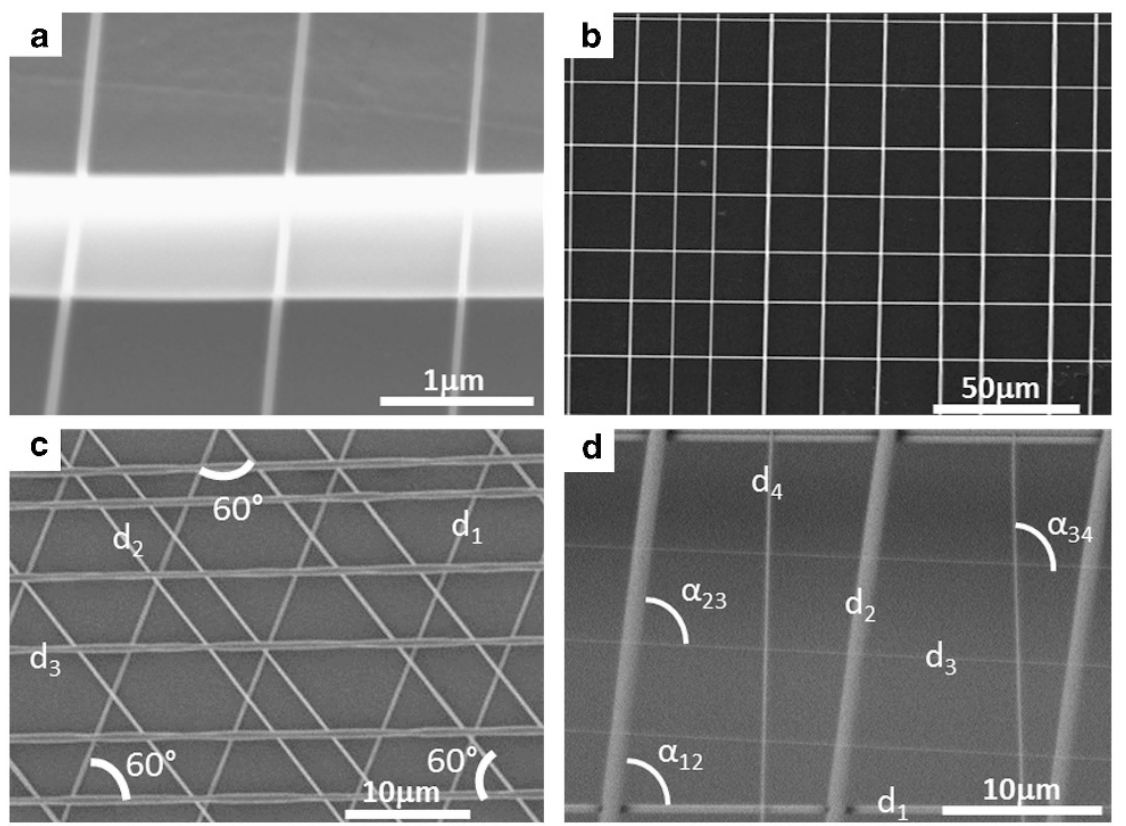

Figure 3 Bottom-up PS nanofiber assemblies using STEP: (a) $70 \mathrm{~nm}$ fiber arrays on top of a $800 \mathrm{~nm}$ diameter fiber; (b) $800 \mathrm{~nm}$ diameter fiber arrays in orthogonal configurations; (c) three layers of fiber arrays configured at $60^{\circ}$ angles with each other with $d_{1}=d_{2}=400 \mathrm{~nm}, d_{3}=800 \mathrm{~nm}$; and (d) a hierarchical fiber assembly of different diameter fibers with controlled orientations and $d_{1}=400 \mathrm{~nm}$, $d_{2}=800 \mathrm{~nm}, d_{3}=d_{4}=70 \mathrm{~nm} ; \alpha_{12}=\alpha_{23}=80^{\circ}, \alpha_{34}=90^{\circ}$. 
of the polymer chain entanglements necessary to form smooth and uniform fibers. In Equations (5)-(7), the decreasing exponents demonstrate the diminishing role of molecular weight in the fiber formation process as the concentration increases, which can be explained through the relationship between the gyration radius of the polymer chain $\left(R_{\mathrm{G}}\right)$ and the polymer solution concentration $(C)$ : $\mathrm{R}_{\mathrm{G}} \sim C^{-1 / 4} .^{26}$ An increase in the polymer solution concentration makes the polymer chains in solution more compact, thus limiting the effect of the molecular weight.

The role of increased polymer chain entanglements with an increase in (i) the molecular weight (at the same concentration) and (ii) the concentration (at the same molecular weight) is to restrict the ability of individual polymeric chain segments to undergo physical deformation in the extensional stress field during the fiber formation process. The scaling laws developed for the fiber diameter control (Equations (1)-(7)) are mapped to obtain the Isodiametric design space (Figure 2c). Through visual inspection of the isodiameter lines (blue lines) in the mapped design space, determining the regions of solution rheology with sufficient polymer chain entanglements for the fabrication of smooth, uniform fibers with diameters ranging from 70 to $800 \mathrm{~nm}$ and lengths of at least several millimeters is straightforward. The STEP-based uniform diameter fiber arrays were obtained at concentrations closer to and greater than $C_{\mathrm{e}}$ (Supplementary Information, SI Table 1), which indicates the transition from the semi-dilute unentangled domain to the semi-dilute entangled domain. At $C_{\mathrm{e}}$, the molecular chains are considered to have sufficient overlaps, thus forming a long-range physical entanglement network, which is necessary for the continuous fabrication of smooth and uniform fibers. An increase in the concentration beyond $C_{\mathrm{e}}$ leads to compacted polymeric chains due to a decreased number of solvent molecules entrapped in the entangled polymer chain networks, which ultimately results in larger diameters under the same external extensional stress.

The limiting cases for the formation of fibers with acceptable attributes (smooth, defect-free and of substantial length) occur at solution rheologies with very high and very low concentrations. At low concentrations (semi-dilute un-entangled and dilute domains), there are not enough polymer molecules to form long-range networks, yielding the classic beaded morphology as shown in insert 1 (corresponding to point 1 in the design space). At the other extreme of highly concentrated rheologies, the absence of solvent molecules leads to the convergence of isodiameter lines, indicating the limits of dry-spinning-based manufacturing methods, such as STEP and electrospinning. At these high concentrations, fibers of large diameters (tens of microns) were obtained along with fiber beads, as shown in insert 4 (corresponding to point 4 in the design space); these fibers are comparable to those obtained from traditional melt-spinning manufacturing methods. ${ }^{28-30}$

Fibers of increasing diameters were obtained through the transition of solution rheologies from semi-dilute to concentrated regimes. As shown in Figure $2 \mathrm{c}$ inserts 2 and 3, aligned $70 \mathrm{~nm}$ diameter fiber arrays were obtained in the semi-dilute entangled region (point 2), while $500 \mathrm{~nm}$ diameter fiber
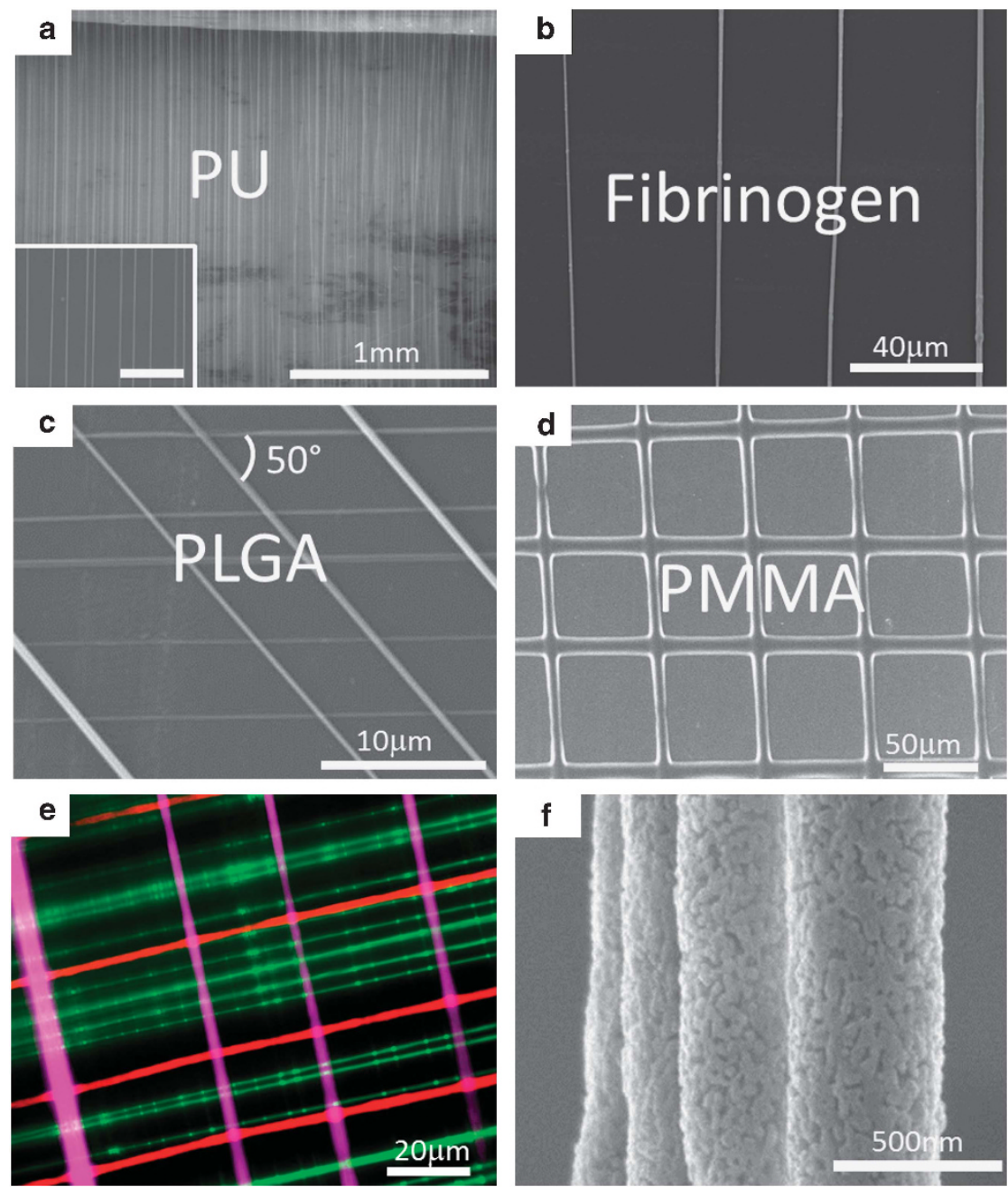

Figure 4 Fiber assemblies made from various materials: (a) suspended unidirectional PU fibers with the scale bar in the insert representing $25 \mu \mathrm{m}$; (b) evenly spaced unidirectional fibrinogen fibers; (c) a PLGA fiber assembly with top and bottom layers configured at a $50^{\circ}$ angle; (d) an inter-connected orthogonal PMMA fiber network; (e) an assembly incorporating PU fibers (green), PS (red) and PLGA fibers (purple); and (f) an array of densely packed uniform diameter titanium dioxide fibers. 
inter-fiber spacing was difficult to control. In contrast to these approaches, STEP is capable of generating nanofiber assemblies with unique control of the fiber dimensions, spacing and orientations. Uniform diameter STEP fibers are configured in parallel arrays as they are collected on substrates, whereas the space between fibers is easily controlled by adjusting the substrate translation speed. At constant rotational speeds, the fiber spacing is directly proportional to the translation speed. In addition, layers of fiber arrays can be deposited on top of previously deposited layers at any desired angle. Owing to the absence of an electric field, sub- $100 \mathrm{~nm}$ diameter fibers can be easily deposited in the form of evenly spaced uniform fiber arrays without being disturbed by any spinning instabilities. In Figures $3 a$ and b, we demonstrate $70 \mathrm{~nm}$ diameter fiber arrays on top of an $800 \mathrm{~nm}$ diameter fiber and a crisscross fiber assembly of $800 \mathrm{~nm}$ diameter fibers forming a matrix of rectangular unit cells. Notice that from Figures $3 \mathrm{a}$ to $3 \mathrm{~b}$, the fiber spacing was increased from 1 to $16 \mu \mathrm{m}$ by increasing the translational speed from 0.02 to $0.32 \mathrm{~mm} \mathrm{~s}^{-1}$. More complex fiber patterns were achieved by stacking several layers of fiber arrays together while varying the interlayer angles. Figure $3 c$ presents an assembly formed by three layers of fiber arrays (diameters: $d_{1}=d_{2}=400 \mathrm{~nm}, \quad d_{3}=800 \mathrm{~nm}$ ) deposited at $60^{\circ}$ angles to each other, thereby creating equilateral triangles. In addition, large diameter fiber networks can be used as the basis of the assembly, over which sub$100 \mathrm{~nm}$ diameter fiber networks form the top structures, resulting in a bottom-up hierarchical structure. Figure 3d shows such a hierarchical assembly, which incorporates a basis made of medium diameter fibers $\left(d_{1}=400\right.$ $\mathrm{nm})$ and large diameter fibers $\left(d_{2}=800 \mathrm{~nm}\right)$ and a top structure made of small diameter fibers $\left(d_{3}=d_{4}=70 \mathrm{~nm}\right)$ with controlled orientations $\left(\alpha_{12}, \alpha_{23}, \alpha_{34}\right)$ and fiber spacing.

The proposed STEP-based methodology is able to achieve nano/micro fiber arrays of various polymer species with control of the fiber diameter, spacing and orientation. Figure 4a presents a suspended unidirectional polyurethane (PU) fiber array (diameter: $\sim 450 \mathrm{~nm}$ ), whereas Figures $4 \mathrm{~b}$ and c present fibrinogen fiber (diameter: $400-900 \mathrm{~nm}$ ) and PLGA fiber (diameter 150-500 nm) assemblies, respectively. Figure $4 \mathrm{~d}$ shows an interconnected PMMA fiber network composed of $3 \times 4$ equal size unit cells that was obtained by treating the crisscross PMMA fiber assembly with solvent vapor. The PMMA fibers became flat ribbons of $4-5 \mu \mathrm{m}$ in width and were fused at intersections. Uniquely, the fibers of different species can be incorporated into a single assembly as shown in Figure 4e, where a suspended three-layer assembly is composed of three species of polymer fibers: a top layer of PLGA fibers (purple), a middle layer of PS fibers (red) and a bottom layer of PU fibers (green).

In addition, composite nanofibers consisting of inorganics and polymers can be fabricated by spinning solutions containing both PS and metal alkoxide. Calcination of aligned composite nanofibers at elevated temperature removes PS, resulting in pure ceramic fibers as indicated by the array of densely packed uniform diameter titanium dioxide fibers (diameter: $\sim 400 \mathrm{~nm}$ ) in Figure $4 \mathrm{f}$. The development of precise inorganic fiber assemblies is part of our future work.

\section{CONCLUSION}

An isodiametric design space is developed to deposit PS fibers with diameters ranging from 70 to $800 \mathrm{~nm}$ with lengths of at least several millimeters. Molecular entanglementbased scaling laws are used to investigate the role of polymer chain entanglements on fiber formation and to demonstrate that smooth, uniform fibers of substantial length are obtained at concentrations approaching and exceeding the critical entangled concentration $\left(C_{\mathrm{e}}\right)$. Visual inspection of the isodiametric design space provides knowledge of the polymer molecular weight and solution concentration rheology required to fabricate continuous high aspect ratio fibers. The design space-based assembly of fibers in three dimensions with control of the fiber dimensions and spatial deposition (including orientation and spacing) is demonstrated for multiple polymer species. The design framework outlined in this study is generalizable to inorganic material systems and establishes a much needed design framework for the highly repeatable and controllable deposition of isodiametric micro/nanofibers in a bottom-up assembly environment.

\section{ACKNOWLEDGEMENTS}

We thank Steve McCartney for his help with scanning electron microscope imaging. We thank Kevin Sheets, Puja Sharma, Sean Meehan and Colin Ng for helpful discussion. We are also grateful to Institute for Critical Technologies and Applied Sciences (ICTAS) and the Nanoscale Characterization and Fabrication Laboratory (NCFL), ICTAS at VT.

$$
\begin{gathered}
\text { Amrinder S Nain }{ }^{1,2,3,5} \text { and Ji Wang }{ }^{3,4,5} \\
{ }^{1} \text { Department of Mechanical Engineering, } \\
\text { Virginia Tech, Blacksburg, VA, USA; }
\end{gathered}
$$

${ }^{2}$ School of Biomedical Engineering and Sciences, Virginia Tech, Blacksburg, VA, USA;

${ }^{3}$ Macromolecules and Interfaces Institute, Virginia Tech, Blacksburg, VA, USA and ${ }^{4}$ Department of Engineering Science and Mechanics, Virginia Tech, Blacksburg, VA, USA E-mail: nain@vt.edu ${ }^{5}$ These authors contributed equally to this work.

1 Nanjundappa, R. \& Bhat, G. Effect of processing conditions on the structure and properties of polypropylene spunbond fabrics. J. Appl. Poly. Sci. 98, 2355-2364 (2005).

2 Ariawan, A. B., Hatzikiriakos, S. G., Goyal, S. K. \& Hay, H. Effects of molecular structure on the rheology and processability of blow-molding high-density polyethylene resins. Adv. Polym. Technol. 20, 1-13 (2001).

3 Zhao, R. \& Wadsworth, L. C. Study of polypropylene/ poly(ethylene terephthalate) bicomponent melt-blowing process: The fiber temperature and elongational viscosity profiles of the spinline. J. Appl. Poly. Sci. 89, 1145-1150 (2003).

4 Ziabicki, A. Fundamentals of Fiber Formation (Wiley, New York, 1976).

5 Ohzawa, Y., Nagano, Y. \& Matsuo, T. J. Studies on dry spinning. I. Fundamental equations. J. Appl. Polym. Sci. 13, 257-283 (1969).

6 Rwei, S. P., Jue, Z. F. \& Chen., F. L. PBT/PET conjugated fibers: melt spinning, fiber properties, and thermal bonding. Polym. Eng. Sci. 44, 331-344 (2004).

7 Suzuki, A. \& Kishi, M. Preparation of poly(ethylene terephthalate) nonwoven fabric from endless microfibers obtained by $\mathrm{CO} 2$ laser-thinning method. Polymer 48, 2729-2736 (2007).

8 Reneker, D. H. \& Chun, I. Nanometre diameter fibres of polymer, produced by electrospinning. Nanotechnology 7, 216-223 (1996).

9 Reneker, D. H., Yarin, A. L., Fong, H. \& Koombhongse., S. Bending instability of electrically charged liquid jets of polymer solutions in electrospinning. J. Appl. Physics 87, 4531-4547 (2000).

10 Doshi, J. \& Reneker, D. H. Electrospinning process and applications of electrospun fibers. J. Electrostatic. 35, 151-160 (1995).

11 Theron, A., Zussman, E. \& Yarin, A. Electrostatic fieldassisted alignment of electrospun nanofibres. Nanotechnology 12, 384-390 (2001).

12 Zussman, E., Theron, A. \& Yarin, A. L. Formation of nanofiber crossbars in electrospinning. Appl. Phys. Lett. 82, 973-975 (2003).

13 Martin., C. R. Nanomaterials: a membrane-based synthetic approach. Science 266, 1961-1966 (1994).

14 Feng, L., Li, S., Li, H., Zhai, J., Song, Y., Jiang, L. \& Zhu, D. A. Super-hydrophobic surface of aligned polyacrylonitrile nanofibers. Angew. Chem. Int. Ed. 41, 1221-1223 (2002).

$15 \mathrm{Ma}$, P. X. \& Zhang, R. J. Synthetic nano-scale fibrous extracellular matrix. Biomed. Mater. Res. 46, 60-72 (1999).

16 Yang, F., Murugan, R., Ramakrishna, S., Wang, X., Ma, Y. X. \& Wang, S. Fabrication of nano-structured porous PLLA scaffold intended for nerve tissue engineering. Biomaterials 25, 1891-1900 (2004).

17 Nain, A. S., Amon, C. \& Sitti, M. Proximal probes based nanorobotic drawing of polymer micro/nanofibers. IEEE Trans. Nanotechnol. 5, 499-510 (2006).

18 Nain, A. S., Wong, J. C., Amon, C. \& Sitti, M. Drawing suspended polymer micro-/nanofibers using glass micropipettes. Appl. Phys. Lett. 89, 183105 183105-3 (2006).

19 McKee, M., Wilkes, G. L., Colby, R. H. \& Long, T. E. Correlations of solution rheology with electrospun fiber formation of linear and branched polyester. Macromolecules 37, 1760-1767 (2004). 
20 Shenoy, S. L., Bates, W. D., Frisch, H. L. \& Wnek, G. E. Role of chain entanglements on fiber formation during electrospinning of polymer solutions: good solvent, non-specific polymer-polymer interaction limit. Polymer 46, 3372-3384 (2005).

21 Gupta, P., Elkins, C., Long, T. E. \& Wilkes, G. L. Electrospinning of linear homopolymers of poly(methyl methacrylate): exploring relationships between fibe formation, viscosity, molecular weight and concentration in a good solvent. Polymer 46, 4799-4810 (2005).

22 Lee, K. H., Kim, H. Y., Bang, H. J., Jung, Y. H. \& Lee., S. G. The change of bead morphology formed on electrospun polystyrene fibers. Polymer 44, 4029 4034 (2003)

23 Wang, C., Hsu, C. H. \& Lin, J. H. Scaling laws in electrospinning of polystyrene solutions. Macromolecules 39, 7662-7672 (2006)

24 Carnell, L. S., Siochi, E. J., Holloway, N. M., Stephens, R. M., Rhim, C., Niklason, L. E. \& Clark, R. L. Aligned mats from electrospun single fibers. Macromolecules 41, 5345-5349 (2008)
25 Nain, A. S., Sitti, M., Jacobson, A., Kowalewski, T. \& Amon., C. Dry spinning based spinneret based tunable engineered parameters (STEP) technique for controlled and aligned deposition of polymeric nanofibers. Macromol. Rapid Commun. 30, 1406-1412 (2009).

$26 \mathrm{Heo}$, Y. \& Larson., R. G. The scaling of zero-shear viscosities of semidilute polymer solutions with concentration. J. Rheol. 49, 1117-1128 (2005).

27 Graessley, W. W. Polymer chain dimensions and the dependence of viscoelastic properties on concentration, molecular weight and solvent power. Polymer 2, 258-262 (1980).

28 Spruiell, J. E. \& White, J. L. Structure development during polymer processing: studies of the melt spinning of polyethylene and polypropylene fibers. Polym. Eng. Sci. 15, 660-667 (1975).

29 Young, W. K. \& Chang, D. H. Studies on melt spinning. VII. elongational viscosity and fiber morphology of multiphase polymer systems. J. Appl. Poly. Sci. 21, 515-524 (1977).
30 Dees, J. R. \& Spruiell, J. E. Structure development during melt spinning of linear polyethylene fibers. J. Appl. Poly. Sci. 18, 1053-1078 (1974).

31 Sarkar, S., Deevi, S. \& Tepper., G. Biased AC electrospinning of aligned polymer nanofibers. Macromol. Rapid Commun. 28, 1034-1039 (2007).

32 Chang, C., Limkrailassiri, K. \& Lin, L. Structure development during polymer processing: studies of the melt spinning of polyethylene and polypropylene fibers. Appl. Phys. Lett. 82, 123111-123113 (2003).

33 Ding, Z., Salim, A. \& Ziaie, B. Selective nanofiber deposition through field-enhanced electrospinning. Langmuir 25, 9648-9652 (2009).

34 Harfenist, S. A., Cambron, S. D., Nelson, E. W., Berry, S. M., Isham, A. W., Crain, M. M., Walsh, K. M., Keynton, R. S. \& Cohn, R. W. Direct drawing of suspended filamentary micro- and nanostructures from liquid polymers. Nano Lett. 4, 1931-1937 (2004).

35 Badrossamay, M. R., Mcilwee, H. A., Goss, J. A. \& Parker, K. K. Nanofiber assembly by rotary jet-spinning. Nano Lett. 10, 2257-2261 (2010).

Supplementary Information accompanies the paper on Polymer Journal website (http://www.nature.com/pj) 